\title{
ESTUDIO DE LAS PROPIEDADES TERMOFISICAS DE CONGELACION DEL MUSCULO DE CAMARON GIGANTE (Macrobrachium rosenbergii 2. Contenido de hielo y Calor específico aparente
}
F. Coronado J. *
O. M endieta T. *
F. Kleeberg $\mathrm{H}$.

\section{RESUMEN}

En el presente trabajo los valores de peso molecular efectivo de sólidos, contenido y velocidad de formación de hielo y calor específico aparente de músculo de camarón gigante, son estimados empleando las ecuaciones propuestas por Schwartzberg (1976) y Chen (1985); a partir de datos experimentales de punto inicial de congelación y ental pia.

Los valores predecidos por ambos modelos, para las propiedades termofísicas mencionadas anteriormente, no presentan diferencias significativas.

Palabras claves: Camarón gigante, congelación, calor específico aparente.

\section{INTRODUCCION}

El camarón gigante $M$ acrobrachium rosenbergii es un crustáceo, natural de la región Indopacífica, de alto valor alimenticio y económico, que ha sido introducido recientemente en la región San Martín y se proyecta que en un futuro cercano alcanzará altos niveles de producción. Siendo la congelación el método de conservación más utilizado para el camarón, se considera de importancia conocer las propiedades termofísicas del mismo,

El objetivo del presente trabajo fue estimar el peso molecular efectivo, porcentaje y velocidad de formación de hielo con la temperatura y calor específico aparente, mediante ecuaciones de correlación reportadas en la literatura.

* U niversidad N acional de San M artín-T arapoto. Facultad de Ingeniería A groindustrial. A partado 239. Tarapoto.

** Universidad Nacional A graria L a M olina. Facultad de Ingeniería Pesquera. Lima. 


\section{MATERIALES Y METODOS}

L os datos referentes a punto de congelación y ental pia de congelación para músculo de camarón gigante fueron tomados de un trabajo anterior efectuado por los mismos autores, utilizando muestras cultivadas en estanques en la región San M artín.

La composición química de la materia prima utilizada fue: Humedad, 78.6\%, Proteína, 19.44\%; Grasa, 0.5\% ; Ceniza, 102\% y Carbohidratos, $0.3 \%$.

\section{ME TODOS}

\section{Fracción de masa de agua ligada "b"}

Fue calculada mediante la ecuación (1) Schwartzberg, (1981). Los valores de actividad de agua requeridos para la aplicación de la ecuación (1) fueron determinados reemplazando en la ecuación (2) del descenso crioscópico, las temperaturas iniciales de congelación, obtenidas experimentalmente.

$$
b=\frac{N_{1} A_{w_{2}}-N_{2} A w_{1}+A w_{1} A_{w_{2}}\left(N_{2}-N_{1}\right)}{A w_{2}-A w_{1}}
$$

In $A_{w}=\frac{M_{w} H_{0}}{R}\left|\frac{1}{T_{0}}-\frac{1}{T}\right|$

\section{Relación de pesos moleculares "E"}

Fue obtenida a partir del punto inicial de congelación de la muestra y la aplicación de la ecuación (3). Schwartzberg, (1976).

$$
E=\frac{(N-b)(I-A w)}{A w}
$$

\section{Determinación del peso molecular efectivo "M s"}

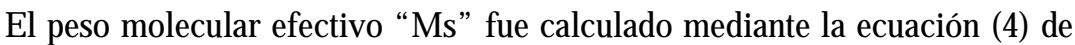
Schwartzberg, H.G. 1976, y también con la ecuación (5) de Chen (5), a partir de valores experimentales de ental pía dentro del rango de $100 \mathrm{C}$ por debajo del punto inicial de congelación de las muestras. 
$M_{8}=18 / E$

$M_{8}=$

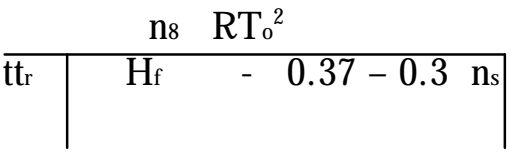

\section{Contenido de hielo}

El contenido de hielo formado a diferentes temperaturas de congelación, expresado como $\mathrm{kg} / \mathrm{kg}$ de alimento fue estimado mediante dos ecuaciones: la ecuación (6) de Schwartzberg, (1976) y la ecuación (7) de Chen, (1985b).

$$
\begin{array}{lrr}
n_{h}=\left(n_{w_{0}}-b n_{s}\right) & \begin{array}{c}
\left(T_{1}-T\right) \\
\left(T_{0}-T\right)
\end{array} \\
n_{h}=n_{s} & R T_{0} 2 & \left(t_{i}-t\right) \\
M s & H_{0} & t_{i}
\end{array}
$$

El contenido de hielo expresado como un porcentaje del contenido inicial de agua fue calculado mediante la ecuación (8)

$$
h=\begin{gathered}
100_{\mathrm{nh}} \\
\mathrm{n}_{\text {wo }}
\end{gathered}
$$

\section{Velocidad de formación de hielo}

Fue estimada usando la ecuación (9) de Schwartzberg, (1976) y la ecuación (10) de chen, (1985b)

$$
\begin{gathered}
S=\text { l }_{w_{0}}-b n_{s} \begin{array}{r}
\left(T_{0}-T_{i}\right) \\
\left(T_{0}-T\right)^{2}
\end{array} \\
S=\begin{array}{llc}
n_{s} & R T_{0} 2 & 1 \\
M_{s} & H_{0} & t_{2}
\end{array}
\end{gathered}
$$




\section{Calor específico aparente}

Fue calculado usando la ecuación (11) de Schwartzberg, (1976) y la ecuación (12) de Chen, (1985h). Para la aplicación de la ecuación (11), el calor específico de la muestra totalmente congelada, Cf fue determinado por la ecuación (13).

$$
\begin{aligned}
& C_{p a}=C_{f}+\left(n_{w o}-b n_{s}\right): \begin{array}{l}
H_{0}\left(T_{0}-T_{i}\right) \\
\left(T_{0}-T\right)^{2}
\end{array} \\
& C_{p a}=0.37+0.3 n_{s}+N_{s} \frac{R T_{0}^{2}}{M_{s}} \overline{t_{2}} \\
& C_{f}=C_{s} n_{s}+C_{n}\left(n_{w o}-b n_{s}\right)+b C_{w} n_{s}
\end{aligned}
$$

\section{RESULTADOS Y DISCUSION}

Los valores obtenidos para los parámetros empíricos de congelación del músculo de camarón de agua dulce fueron los siguientes:

Constante empírica, $\quad \mathrm{b}=0.29$

Peso molecular efectivo, $M_{s}=492.07$

Constante empírica, $\quad \mathrm{E}=0.3658$

El valor de h calculado para el camarón de agua dulce (Macrobrachium rosenbergi) representa la fracción de agua no congelable/ $\mathrm{kg}$. de materia seca, el cual es cercano al valor reportado por Kleeberg, (1986), para pescado crudo y cocido, sin embargo resulta superior al rango de valores indicados por Duckworth (1971), mencionado por Gruda y Postolski (1986), que es de 0.24 a 0.27 .

El peso molecular efectivo, $M$, encontrado para el material en estudio es inferior al reportado para productos similares tales como caracol fresco, Alva, (1990), y pescado crudo, Kleeberg, (1986). Esto se debe al contenido de humedad del camarón fresco que es más elevado que el de los productos mencionados, puesto que el peso molecular efectivo varía en forma inversa con el contenido de humedad.

De otro lado, el valor de $E$ encontrado para el camarón de agua dulce presentará una tendencia creciente similar a los valores de $E$ reportados por 
Kleberg, (1986), para pescado crudo y cocido, dado que E está en relación inversa al peso molecular $\left(\mathrm{M}_{\mathrm{s}}\right)$.

\section{CONTENIDO Y VELOCIDAD DE FORMACION DE HIELO}

L os resultados calculados de contenido y velocidad de formación de hielo a diferentes temperaturas de congelación para músculo fresco de camarón gigante son mostrados en el Cuadro 1. Al pie del cuadro se incluyen los parámetros utilizados para las ecuaciones (5), (6), (9) y (10).

\section{Cuadro 1}

C ontenido y velocidad de formación de hielo calculados en función de la temperatura para camarón gigante

\begin{tabular}{|l|cc|cc|}
\hline \multirow{2}{*}{ o C } & \multicolumn{2}{|c|}{ Contenido de hielo $\mathrm{nh}$} & \multicolumn{2}{c|}{ Velocidad de formación de hielo } \\
& $\mathrm{SCH} / \mathrm{kg})$ & \multicolumn{2}{c|}{$\mathrm{S}\left(\mathrm{kg} / \mathrm{kg}^{\circ} \mathrm{C}\right)$} \\
\hline & & $\mathrm{CHEN}^{2}$ & $\mathrm{SCHW}{ }^{1}$ & $\mathrm{CHEN}^{2}$ \\
0.0 & 0.000 & 0.000 & 0.0000 & 0.0000 \\
-2.3 & 0.377 & 0.383 & 0.1505 & 0.1527 \\
-3.0 & 0.458 & 0.465 & 0.0884 & 0.0897 \\
-4.0 & 0.524 & 0.532 & 0.0497 & 0.0504 \\
-5.0 & 0.564 & 0.572 & 0.0318 & 0.0323 \\
-6.0 & 0.591 & 0.599 & 0.0221 & 0.0224 \\
-7.0 & 0.610 & 0.619 & 0.0162 & 0.0164 \\
-8.0 & 0.624 & 0.633 & 0.0124 & 0.0126 \\
-9.0 & 0.635 & 0.644 & 0.0098 & 0.0099 \\
-10.0 & 0.644 & 0.653 & 0.0079 & 0.0080 \\
-12.0 & 0.657 & 0.667 & 0.0056 & 0.0056 \\
-15.0 & 0.670 & 0.680 & 0.0035 & 0.0035 \\
-20.0 & 0.684 & 0.694 & 0.0019 & 0.0020 \\
-30.0 & 0.697 & 0.707 & 0.0008 & 0.0008 \\
-40.0 & 0.704 & 0.714 & 0.0004 & 0.0005 \\
\hline
\end{tabular}

1 Parámetros empleados: $\mathrm{n}_{\mathrm{wo}}=0.786, \mathrm{~T}_{\mathrm{i}}=-1.1^{\circ} \mathrm{C}, \mathrm{b}=0.29$

2 Parámetros empleados: $\mathrm{n}_{\mathrm{s}}=0.214, \mathrm{~T}_{\mathrm{i}}=-1.1^{\circ} \mathrm{C}, \mathrm{M}_{\mathrm{s}}=492.07$ 
En el cuadro mencionado anteriormente podemos apreciar que los valores calculados mediante las ecuaciones (5) y 6) de Schwartzberg, (1981) y los valores obtenidos mediante las ecuaciones (9) y (10) de Chen, (1985b), no presentan diferencias significativas. Estos resultados son confiables puesto que su precisión para predecir contenidos de hielo en productos cárnicos ha sido demostrada por diferentes investigadores, Schwartzberg, (1981), Chen, (1985b).

Comparando los valores obtenidos para el camarón fresco con valores . consignados en la literatura, para caracol crudo, Alva, (1990), se puede notar que el contenido de hielo a las mismas temperaturas, es mayor para el casco de camarón, lo cual es justificable por que este valor es función del contenido de agua libre. Esto concuerda con los resultados experimentales obtenidos por Riedel (1960), citado por Alva Jorge, (1990), para pescado marino parcialmente deshidratado quien determinó que en muestra con mayor contenido de humedad se formó mayor cantidad de hielo a la misma temperatura.

En cambio, la velocidad de formación de hielo, para las mismas temperaturas consideradas, fue menor en el caso del camarón lo cual se debe a que el punto inicial de congelación de éste es mayor al del caracol crudo.

En la Figura 1, se presenta la curva de contenido de hielo en músculo fresco de camarón gigante obtenida mediante las ecuaciones de Schwartzberg, (1981) y Chen, (1985b). Puede notarse que las curvas se hacen constantes al rededor del $90 \%$ lo cual indica que el resto es agua difícilmente congelable en productos alimenticios, Gruda y Postolski, (1986).

\section{CALOR ESPE CÍFICO APARE NTE}

En la Figura 2 se presenta gráficamente el comportamiento de esta propiedad térmica para músculo de camarón gigante. Puede notarse que los valores obtenidos a través de las ecuaciones de Schwartzberg, (1976) y Chen, (1985b) no presentan diferencias considerables. En ambas curvas, cerca al punto inicial de congelamiento, el calor específico aparente se incrementa a un valor muy alto. La elevación del pico es proporcional al contenido de agua libre e inversamente proporcional al descenso del punto de congelación ( $T_{0}-T_{1}$ ). Esto se debe a la influencia en el desarrollo de las curvas del contenido de agua del producto. 


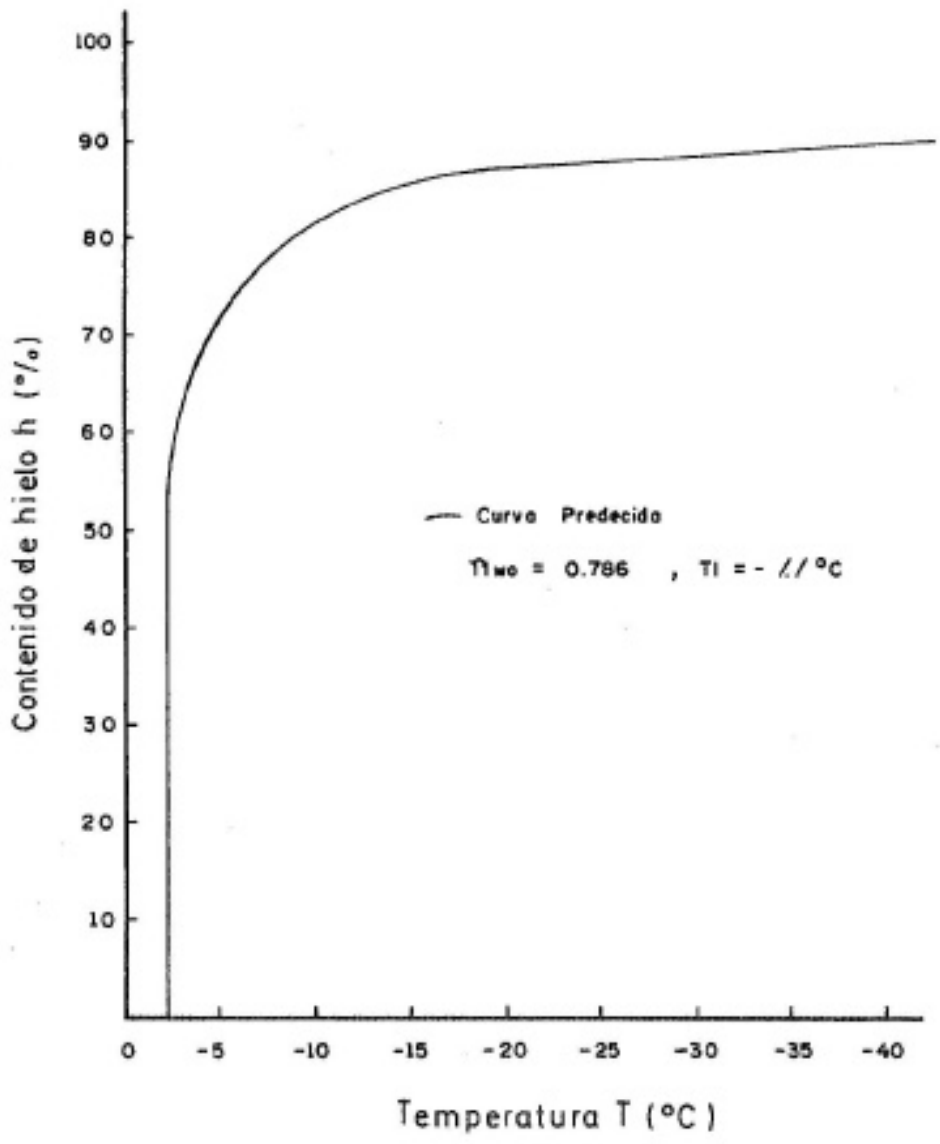

Fig 1 Curva de contenido de hielo predecida para músculo de Camarón gigante. 


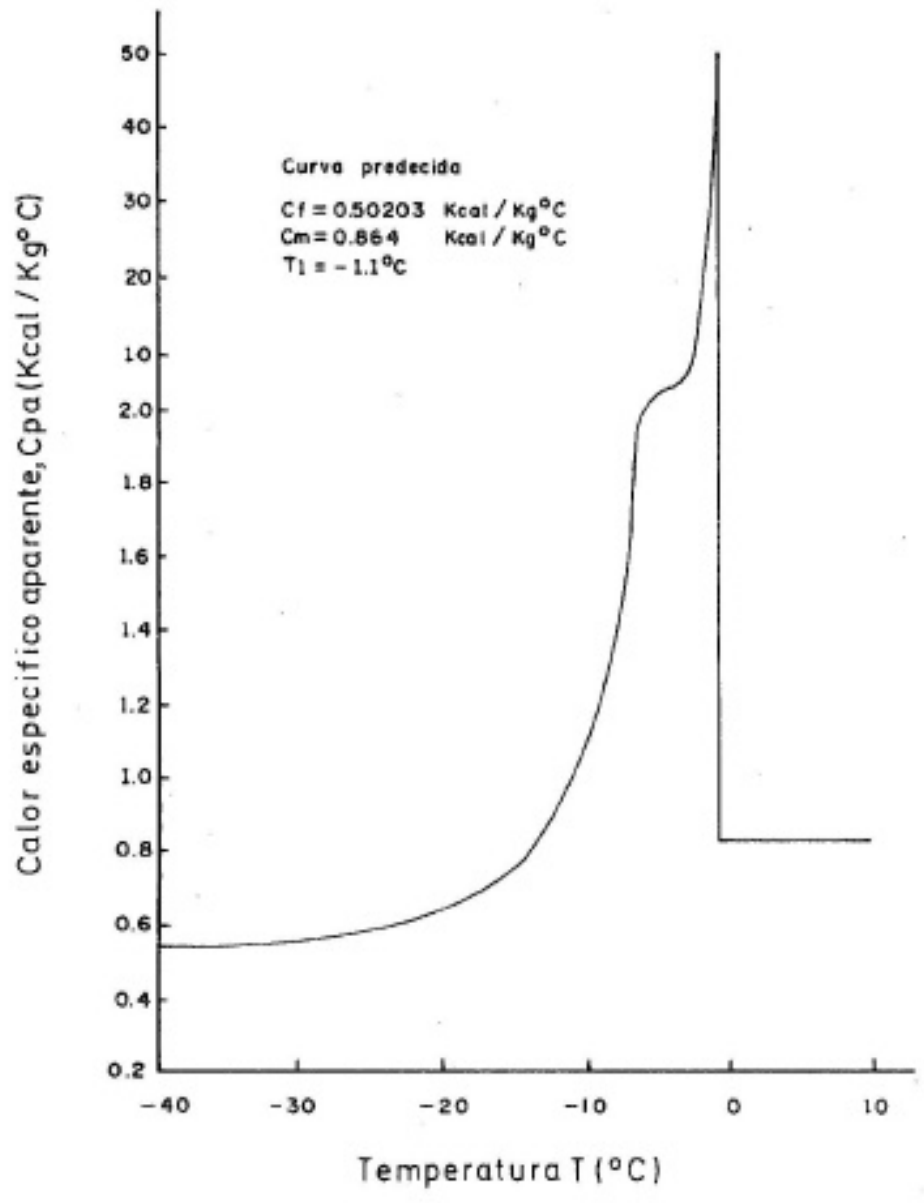

Fig. 2 Curva de calor especifico aparente predecida para músculo de Camarón gigante. 


\section{CONCLUSIONES}

L as principales conclusiones obtenidas en este trabajo son las siguientes:

1. El peso molecular efectivo tiene un valor de $492.07 \mathrm{~kg} / \mathrm{kg}-\mathrm{mol}$.

2. El porcentaje de hielo a -40 o $\mathrm{C}$ es de $89.56 \%$ y $90.84 \%$ mientras que la velocidad de formación de hielo a la misma temperatura es de 0.0004 $\mathrm{kg} / \mathrm{kg} \stackrel{\circ}{ } \mathrm{C}$ y $0.0005 \mathrm{~kg} / \mathrm{kg} \stackrel{\circ}{\mathrm{C}}$ para las ecuaciones de Schwartzberg y Chen respectivamente.

3. El valor del calor específico aparente es de 0.54 y $0.47 \mathrm{kcal} / \mathrm{kg}^{\circ} \mathrm{C}$ para las ecuaciones mencionadas anteriormente.

\section{BIBLIOGRAFIA}

A LV A J., M. 1990. Estudio de los parámetros termodinámicos de congelamiento del caracol (Thais chocolata) precocido. Tesis UNA L M . Lima- Perú.

A.O.A.C. 1970. Official M ethods of A nalysis A ssociation of Official A griculture Chemist, 1 lava Edición.

BARRAL, A.0., N.J. BERGAMASHI y C.A. CASTANON, 1986. Planillas de puntaje organoléptico para langostino (Pleoticus muelleri) fresco y congelado. FAO Informe de Pesca NO 421. Suplemento. Santiago de Chile.

CHEN, C.S. 1985a. Thermodynamic analysis of the freezing and thawing of foods: Enthalpy and A pparent Specific Heat. J. Food Sci. 50: 1158.

CHEN, C.S. 1985b. Thermodynamic analysis of the freezing and thawing of foods: Ice content and M ollier Diagram, J. Food Sci. 50: 1163-1166,

GRUDA, Z. y J. POSTOLSKI, 1986. Tecnología de la congelación de los alimentos. Edit. Acribia S.A. 631.

HSIEH, L.E. LEREW y DR. HELDMAN, 1977. Prediction of freezing times for foods as influeced by product properties. J.. Food Process Engineering. Vol. 1. 183. 
KLEEBERG, H.F. 1986. Estudio comparativo dos parametros termodinamicos e da cinetica de congelamento de file de pescado (Cynoscion petranas) cru e cozido. Tesis. Universidade de Gradual de Campinas. Sáo Paulo.

NEW, B.M. y 5. SINGHOLKA, 1984. Cultivo de camarón de agua dulce. $M$ anual para el cultivo de Macrobrachium rosenbergii. Documento Técnico de Pesca (225): 118 p.

REDDY, NIP K.W. and TANG. 1981. Changes in fatty acids and sensory quality of fresh water prawn (Macrobrachium rosenbergii) stored under frozen conditions. J. Food Sci.46: 353-356

SANTOS, M.R. 1986. Manual de procesamiento y control de calidad de langostinos congelado. Planta Piloto D pto. de Congelado. $\mathrm{N} \cong 2$ 2, Año II. Comisión Editorial Publicaciones. Callao - Peru.

SCHWARTZBERG, H.G. 1976. Effective heat capacities for the freezing and thaw mg of food J. Food Sci. 41:152-156.

SCHWARTZBERG, H.G. 1981. Mathematical Analysis of the Freezing and Thawing of F oods. AICHE. Summer M eeting, A ugust Detroit. USA.

WHEATON F. and 1. LAWSON 1985. Processing Aquatic Food Products. Ed. Jhon Wiley $\&$ Sons. USA. 


\section{ANEXO 1 \\ NOMENCLATURA UTILIZADA}

$A_{w} \quad=$ Actividad de agua

a $=$ Porcentaje de contenido de agua

b = = Fracción de "agua ligada" ( $\mathrm{kg} / \mathrm{kg}$ sólidos)

$C_{p}, C_{c}=$ Calor específico

$\mathrm{C}_{\mathrm{pa}}=$ Calor específico aparente $(\mathrm{kcal} / \mathrm{kg} \cong \mathrm{C})$

$\mathrm{C}_{\mathrm{f}}=$ Capacidad cal orífica de la muestra totalmente congelada ( $\mathrm{kcal} / \mathrm{kg} \cong \mathrm{K}$

$\mathrm{C}_{\mathrm{s} n} \mathrm{~s}_{\mathrm{s}}=$ Calor específico de los sólidos totales expresado en $\mathrm{Kcal} / \mathrm{kg}$.

A limento $\stackrel{\circ}{C}$

$C_{s}, C_{w}=C_{w(t)}, C_{h(t)}=$ Calor específico de la porción sólida, del agua, del agua no congelada a temperatura de congelación y del agua congelada del alimento, que puede no ser dependiente de la temperatura si el calor específico del hielo es respectivamente. ( $\mathrm{K} \mathrm{cal} / \mathrm{kg} \cong \mathrm{C}$ )

$\mathrm{C}_{\mathrm{m}}=\mathrm{C}$ alor asociado de la muestra ( $\mathrm{Kcal} / \mathrm{kg}$ alimento $\left.\cong \mathrm{C}\right)$

$\mathrm{H}=$ Entalpía de congelación $(\mathrm{Kcal} / \mathrm{kg})$

$\mathrm{H}_{0}=$ Calor latente de fusión del agua $(79.8 \mathrm{Kcal} / \mathrm{kg})$

$\mathrm{H}_{\mathrm{f}} \quad=$ Ental pía por debajo de la congelación ( $\mathrm{Kcal} / \mathrm{kg}$ )

$\mathrm{H}=$ Contenido de hielo $(\%)$

$\mathrm{M}_{\mathrm{w}} \quad=$ Peso molecular del agua pura $(18 \mathrm{~kg} / \mathrm{kg}-\mathrm{mol})$

$n_{w}=$ Fracción de agua no congelada a la temperatura de congelación $(\mathrm{kg} / \mathrm{kg})$ de alimento

$\mathrm{n}_{\mathrm{s}} \quad=$ Fracción de sólidos totales (solubles e insolubles, $\mathrm{Kg} / \mathrm{kg}$ del alimento

nwo $=$ Fracción inicial de agua al alimento $(\mathrm{kg} / \mathrm{kg}$ alimento)

$\mathrm{n}_{\mathrm{h}}=$ Fracción de hielo $(\mathrm{kg} / \mathrm{kg}$ de alimento) 
$q_{c}=$ Calor asociado con la capacidad térmica del calorímetro entre las temperaturas inicial final (K cal)

$\mathrm{R}=$ Error medio relativo

$\mathrm{S}=$ V elocidad de formación de hielo $(\mathrm{kg} / \mathrm{kg}$ de al. ㅇ C $)$

ó $\mathrm{kg}$ de masa de hielo formado por grados de descenso en la temperatura por $\mathrm{kg}$ de alimento.

$\mathrm{T}, \mathrm{t}=$ Temperatura a temperatura de congelación

To $\quad=$ Temperatura del punto de congelación de la gua $(273.150 \mathrm{~K})$

$\mathrm{T}_{\mathrm{i}}, \mathrm{t}_{\mathrm{i}}=$ Temperatura inicial de congelación $(\underline{0} \mathrm{C}, \underline{0} \mathrm{~K})$

tr $=$ Temperatura de referencia

two $=$ Temperatura inicial del agua $(\underline{0} \mathrm{C})$

tc $=$ Temperatura de equilibrio

$\mathrm{W}_{\mathrm{w}} \quad=$ Peso del agua

$\mathrm{W}_{\mathrm{m}} \quad=$ Peso de la muestra del camarón gigante $(\mathrm{kg})$

$0.20=$ Base arbitraria del calor específico de los constituyentes sólidos. 